\title{
Impacts of glyphosate-based herbicides on disease resistance and health of crops: a review
}

Daisy A. Martinez ${ }^{1}$, Ulrich E. Loening ${ }^{2^{*}}\left(\mathbb{D}\right.$ and Margaret C. Graham ${ }^{3}$

\begin{abstract}
Based on experimental data from laboratory and field, numerous authors have raised concern that exposure to glyphosate-based herbicides (GBHs) may pre-dispose crops to damage by microbial pathogens. In this review, we distinguish and evaluate two principal pathways by which GBHs may affect the susceptibility of crops to disease: pathway 1-via disruptions to rhizosphere microbial ecology, and pathway 2-via restriction of nutrients to crops. We conclude that GBHs have the potential to undermine crop health in a number of ways, including: (i) impairment of the innate physiological defences of glyphosate-sensitive (GS) cultivars by interruption of the shikimic acid pathway; (ii) impairment of physiological disease defences has also been shown to occur in some glyphosate-resistant (GR) cultivars, despite their engineered resistance to glyphosate's primary mode of action; (iii) interference with rhizosphere microbial ecology (in particular, GBHs have the potential to enhance the population and/or virulence of some phytopathogenic microbial species in the crop rhizosphere); and finally, (iv) the as yet incompletely elucidated reduction in the uptake and utilisation of nutrient metals by crops. Future progress will best be achieved when growers, regulators and industry collaborate to develop products, practices and policies that minimise the use of herbicides as far as possible and maximise their effectiveness when used, while facilitating optimised food production and security.
\end{abstract}

Keywords: Glyphosate-based herbicides, Rhizosphere, Plant disease, Soil microbes

\section{Introduction}

Since its commercial introduction in 1974 as the active ingredient of the broad-spectrum herbicide 'Roundup ${ }^{\circledR}$, glyphosate ( $N$-(phosphonomethyl)glycine) has rapidly become the most extensively used herbicide in the history of agriculture [1-4]. Glyphosate-based herbicides (GBHs) work by blocking the activity of the enzyme 5-enolpyruvylshikimate-3-phosphate synthase (EPSPS) in the shikimic acid pathway used by plants for the biosynthesis of aromatic amino acids. Disruption of the pathway prevents their synthesis, causing plant death by amino acid starvation. GBHs are purported to offer several agricultural and municipal benefits, including:

\footnotetext{
*Correspondence: vel@loening.com

${ }^{2}$ Ormiston Hall, Formerly Centre for Human Ecology and Department of Zoology, University of Edinburgh, EH35 5NJ Edinburgh, Scotland, UK Full list of author information is available at the end of the article
}

(i) Broad-spectrum, systemic weed control GBHs are effective in the control of a very wide range of plant species, including annual and perennial broadleaves and grasses, aquatic vegetation and a number of invasive species common in agricultural and amenity environments [5]. Glyphosate is rapidly translocated from leaves throughout all plant tissues and experiences little or no within-plant degradation.

(ii) Enabling conservation tillage GBHs are used widely in 'no-till' or 'zero-till' systems for pre-sowing and post-harvest 'burndown' or 'knockdown' applications. These applications are for herbicidal removal of a cover crop or of unwanted crop remains before planting, a practice which reduces the need for tillage, thus reducing contributions to tillage-associated soil compaction, erosion and nutrient depletion, and allowing growers to sow seeds beneath a protective cover or 'mulch' of decaying plant material. 
(iii) Specificity Due to the specificity of their intended mode of action (glyphosate inhibits the activity of a single enzyme which is essential in all plants and in some microorganisms, but not in animals), some researchers consider GBHs to be relatively toxicologically benign. Many studies also report short half-lives in soil (attributed to rapid microbial degradation) and limited bioavailability and/or transport in soil due to strong sorption onto soil mineral surfaces $[1,5,6]$. As a consequence of this specificity and effectiveness, GBHs are also used extensively in urban areas for weed control on roadsides and in public parks, etc.

Although there are clear potential benefits from use in certain settings, a number of potentially deleterious side effects of GBHs are emerging. The areas of concern include the possibility that GBH use may lead to an increased susceptibility of crops to damage by microbial pathogens. In this article, we review field and laboratory experimental work reported in the literature concerning the effects of GBHs on the susceptibility of crops to disease. Specifically, our aims were to: (i) draw together current knowledge about the impacts of GBHs on crop health, (ii) distinguish and evaluate the principal ways in which GBHs may affect the susceptibility of crops to disease, (iii) identify key gaps in current understanding and (iv) make recommendations for future research directions.

We have endeavoured to select pertinent experimental studies that, taken together, build a consistent account of the principal ways in which GBHs may interfere with crops' disease-resistance. We discuss first the key properties of glyphosate and GBHs, and their fate in plants and soils. We then evaluate experimental findings concerning herbicide-induced changes to the rhizosphere microbial community, as well as discussing the potential impacts of such changes upon crop health. Following this, we review the currently limited findings concerning the impacts of GBHs on crop mineral nutrition.

\section{Properties of glyphosate}

\section{Chemical structure and mode of action}

The glyphosate molecule has three active groups: carboxylic acid (pK1 $=2.23)$, phosphonate $(\mathrm{pK} 2=5.46)$ and amine (pK3 =10.14) groups (Fig. 1a) and is produced, for example, by the reaction of the amino acid glycine with paraformaldehyde and dimethyl phosphonate. At $\mathrm{pH}$ below 2.23, glyphosate has a net zero charge, since the amino group carries one positive charge and the phosphonate group already has one negative charge; in the $\mathrm{pH}$ range 2.23-5.46, the carboxyl group dissociates and the molecule carries a net negative charge of one; above $\mathrm{pH}$

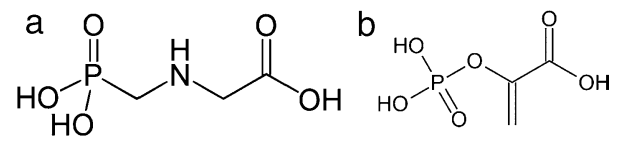

Fig. 1 Molecular structures of $\mathbf{a}$ glyphosate and $\mathbf{b}$ phosphoenolpyruvic acid

5.46, the phosphonate group loses its second proton, the molecule then has a net negative charge of two; above $\mathrm{pH}$ 10.14 , the amine loses its proton and the glyphosate molecule then has a net negative charge of three. The physical, chemical and toxicological properties of glyphosate are given in a technical fact sheet produced by the US National Pesticide Information Centre [7].

These three functional groups, $\mathrm{PO}_{4}{ }^{3-}, \mathrm{COO}^{-}$and $\mathrm{R} 2 \mathrm{HNH}^{+}$, are found in many other naturally occurring molecules, but through their synthetic linkage as shown in Fig. 1a they collectively become a powerful biological agent. Glyphosate binds with and inhibits the activity of the enzyme 5-enolpyruvylshikimate-3-phosphate synthase (EPSPS) in plants' shikimic acid pathway. In this pathway, EPSPS catalyses an unusual transfer reaction of the carboxyvinyl portion of phosphoenolpyruvate (PEP, Fig. $1 \mathrm{~b})$ to the $5-\mathrm{OH}$ of shikimate 3-phosphate (S3P), forming EPSP and inorganic phosphate (Pi) (Fig. 2). However, the structure of glyphosate is sufficiently similar to that of PEP (Fig. 1a, b) that it can compete directly with PEP at its binding site, especially so at its binding site in the EPSPS-S3P complex [8] see also [9]. This binding reaction is not metal dependent, but the metal complexes of glyphosate (discussed below) also bind in the same way to EPSPS [10]. Among other effects, inhibition of the shikimate pathway prevents the synthesis of essential aromatic amino acids as well as a host of other aromatic plant products. Therefore, plant death can be at least partially attributed to amino acid starvation throughout all plant tissues.

\section{Metal-binding properties of glyphosate}

In addition to its biological binding action described above, glyphosate is in an almost unique position among herbicides because of its chelating properties [11]. Indeed, it was synthesised as a chelator some 10 years before its herbicidal action was realised. Phosphonates are generally considered to be good metal chelators, but the inclusion of an amine group within the molecule usually increases the binding ability of the phosphonate. All three functional groups can co-ordinate with metal ions, particularly those of transition elements, at near neutral $\mathrm{pH}$ (e.g. Coutinho and Mazo [12], and an earlier study by Glass [13]. Caetano et al. [10] showed how Zn and $\mathrm{Cu}$ bind more extensively to glyphosate than many other 

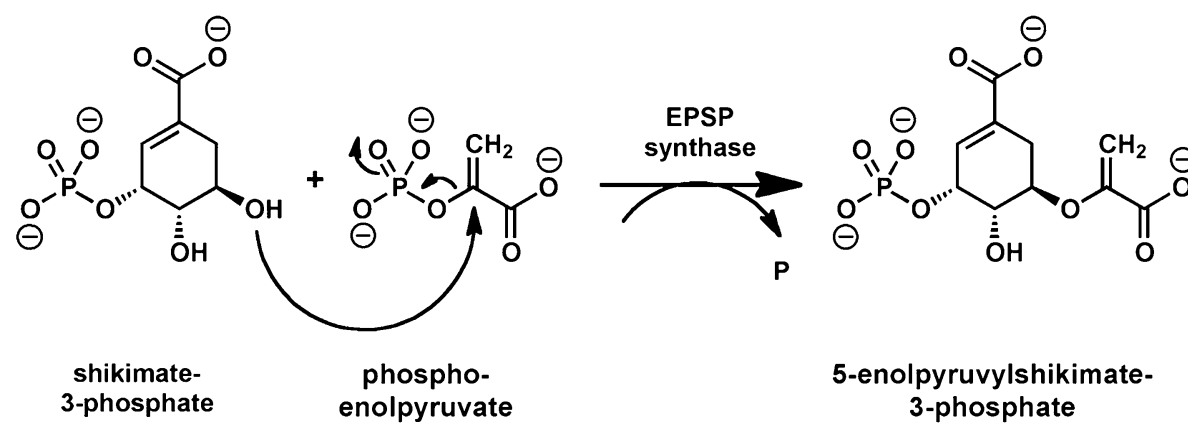

Fig. 2 The shikimic acid pathway

metals. Indeed, copper $(\mathrm{Cu})$ has been shown to form tridentate complexes with two glyphosate molecules per copper atom. More specifically, it is thought that $\mathrm{Cu}^{2+}$ lies at the centre of a Jahn-Teller distorted octahedron with glyphosate forming two five-member rings lying in the equatorial plane [14]. At higher $\mathrm{pH}$, even tetradentate ligands can form if the phosphonate coordinates via two oxygens [11]. In contrast to most phosphonates, however, glyphosate has the unusual property of being easily precipitated out of solution by multivalent metal ions. This may reduce the acute toxic effects associated with certain metals, e.g. $\mathrm{Ag}, \mathrm{Cd}, \mathrm{Cr}, \mathrm{Cu}, \mathrm{Ni}$ and $\mathrm{Pb}$, but some researchers have also reported increased bioaccumulation of others, e.g. Hg in aquatic organisms [15].

Given these chelating properties, it is not surprising that GBHs can exert numerous effects beyond the inhibition of target plants' EPSPS, as will be discussed throughout this review. Potentially any metal-dependent plant metabolic processes could be affected, and thus the wider impacts could be profound.

\section{Distinguishing glyphosate from GBHs}

Several agrochemical companies manufacture GBHs under multiple trade names. These commercial formulations contain various additives, e.g. surfactant petroleum products that facilitate their penetration of plants [16]. Thus, the impacts of GBHs on plants and other organisms may differ substantially from those of glyphosate and its salts, commonly the isopropylamine salt [17-19]. In turn, different GBH formulations have been shown to exert different effects on crops [20]. We endeavour throughout this review to cite the specific formulations used in experiments (e.g. Roundup Ultra Max ${ }^{\circledR}$, Roundup Quick $^{\mathrm{TM}}$ ). Moreover, for impacts of GBHs on crop health to be adequately risk assessed, we recommend that researchers employ commercial formulations rather than pure glyphosate in experimental protocols, since it is these that are relevant in the agricultural context.

\section{The fate of glyphosate in the soil}

Having a systemic action, once applied glyphosate is rapidly translocated throughout plant tissues. Glyphosate residues can accumulate in newly developing plant parts, predominantly in the root and shoot meristematic tissues, but also in belowground reproductive tissues and root nodules [21]. A substantial portion of the residues that accumulate in root tissues will be released to the rhizosphere, whether due to the decay and decomposition of damaged roots or to exudation from the living roots of genetically modified glyphosate-resistant (GR) crops that have been exposed to, but not killed by GBHs [22-25]. Glyphosate applied at sub-lethal doses to non-genetically modified glyphosate-sensitive (GS) crops (where it is used to encourage uniform ripening and to ease harvesting in cereal, grain and oilseed crops) may also be destined for partial exudation from living roots [21, 24]. For example, Kremer et al. [23] reported that when GR soybean plants were treated with Roundup Ultra $^{\circledR}$ at a rate of $0.84 \mathrm{~kg}$ a.e. ha ${ }^{-1}, \sim 1500$ ng glyphosate had been exuded per plant after 16 days. Laitinen et al. [25] reported that 3 weeks after treatment, $8-12 \%$ of the glyphosate applied to the GS plant Chenopodium quinoa had been exuded from roots to the rhizosphere.

Once applied and released from roots, the binding characteristics of glyphosate and its degradation by soil microorganisms are widely reported to limit its persistence in solution and, thus, the potential to cause harm to crops $[1,6,16]$. Thus, Monsanto Company@ advise growers that sowing of a new crop may commence safely as little as $4 \mathrm{~h}$ post-spray treatment with Roundup WeatherMAX ${ }^{\circledR}$ and 24-72 h post-spray treatment with all other Roundup ${ }^{\circledR}$ brand formulations [26]. Detailed evaluations indicate, however, that glyphosate residues released from plant roots cannot be assumed to be entirely or permanently immobilised, or degraded, upon contact with soil. The proportion of glyphosate residues immobilised and/or degraded has been shown to vary 
significantly with local soil composition and properties, as well as with climatic and meteorological conditions, and agronomic practice (reviewed in Borggaard and Gimsing [16]. Likewise, the duration of immobilisation has been shown to be variable, since already-bound glyphosate may be returned to solution (e.g. with the addition of phosphate fertilisers, which may compete with glyphosate for binding sites in soil due to chemical similarity), rendering it available once more for interactions with crops and other non-target organisms [22, 24, $27,28]$ or for leaching and transfer through aquatic environments [29-33].

\section{The influence of GBHs on crops' disease resistance}

The following sections identify and evaluate two principal pathways by which GBHs may interfere with the disease resistance of crops.

\section{Pathway I: disruptions to rhizosphere microbial ecology}

After release from plant roots, glyphosate residues come into contact with an immensely diverse community of microbial species dwelling in the root zone or rhizosphere. Microbial responses to glyphosate vary considerably between species. Some species possess GS forms of microbial EPSPS and suffer metabolic disruptions following exposure to GBHs [34]. Sensitive portions of the ubiquitous rhizosphere biota include some Pseudomonas species and some species of Mn-reducing bacteria [35-37], as well as some species of arbuscular mycorrhizal fungi (AMF) [38-40] and symbiotic N-fixing Bradyrhizobium japonicum bacteroides within soybean nodules [34]. The above are usually regarded as beneficial organisms. It is noteworthy that, in contrast, some potentially phytopathogenic species express relatively glyphosate-tolerant forms of the EPSPS enzyme (including some Fusarium, Pythium and Rhizoctonia spp.) and are unharmed or even stimulated in response to treatment of plants with GBHs $[41,42]$. These differential impacts are of considerable concern, since they may cause alterations to microbial community dynamics that may, in turn, impact negatively upon the health and productivity of crops.

Via its contribution to biogeochemical and nutrient cycling, the soil microbial community as a whole provides a critical service in maintaining soil productivity, sustainability and resilience to perturbations [43]. The following discussion will review key experimental findings concerning herbicide-induced changes to the rhizosphere microbial community, as well as discussing the potential impacts of such changes upon crop health.

\section{Stimulation of phytopathogenic microorganisms}

Phytopathogenic rhizosphere-dwelling microorganisms are found to play a synergistic role in the herbicidal efficacy of GBHs, whereby intensified microbial colonisation on the roots of herbicide-treated plants has been shown to contribute to and/or hasten plant death, in conjunction with the herbicides' intended metabolic mode of action [44-48].

Numerous studies have demonstrated that the population and/or the virulence of certain phytopathogenic species can be enhanced following treatment of plants with GBHs [23, 37, 44, 45, 47-53].

For example, in field studies conducted at multiple sites and over several years (1997-2007), Kremer and Means [37] reported that colonisation of GR soybean roots by three species of Fusarium (Fusarium oxysporum complex, Fusarium solani complex, and Fusarium equiseti complex) was significantly elevated (two- to fivefold) in plants that were treated with a GBH at recommended field rates $\left(0.84 \mathrm{~kg}\right.$ a.e. $\left.\mathrm{ha}^{-1}\right)$, compared with control plants that received no herbicide. Fusarium populations were also consistently elevated in soils where herbicide-treated GR crops had been grown. The authors did not specify the GBH formulation used in their tests. Similarly, when GR soybeans of two cultivars ('BRS242' and 'AG3539') were treated with a GBH (Roundup WeatherMAX ${ }^{\circledR}$; Monsanto, St Louis, MO) at increasing concentrations from $800 \mathrm{~g}$ up to $2400 \mathrm{~g}$ a.e. ha ${ }^{-1}$, Fusarium colonisation of soybean roots increased significantly, in a dose-dependent fashion [36]. In a large-scale field-monitoring programme conducted across multiple cereal-cropping systems in Saskatchewan, Canada, Fernandez et al. [53] assessed the relative importance of various agronomic practices (including tillage and herbicide regimes) as factors determining inoculum levels of fungal pathogens associated with Fusarium head blight (FHB) (including Fusarium avenaceum, Fusarium culmorum and Fusarium graminearum), as well as the prevalence of FHB disease damage in wheat and barley. For both crops, the study identified glyphosate-based weed control methods as the most important management factor associated with elevated Fusarium spp. populations, as well as with increased disease damage in cereal crops year on year.

It has been proposed that proliferations of opportunistic microbial pathogens in the rhizospheres of GBHtreated weeds or crop residues might increase the risk of disease for new crops planted subsequently in the same soil [49-51, 54-56]. Among these, Lynch and Penn [49] found that treatment of quackgrass (Elymus repens) with glyphosate (isopropylamine salt) stimulated rapid colonisation of the roots of this weed by Fusarium culmorum. Enhancement of the rhizosphere F. culmorum population was associated with increased disease damage in barley seedlings planted subsequently in the same soil. An extensive field study conducted by Smiley et al. [50] showed that the inoculum potential for Rhizoctonia 
solani in cereal fields was at its peak 2-3 days after GBH treatment, before gradually declining. Yield depletion and crop damage from Rhizoctonia root rot (caused by Rhizoctonia solani) was most severe when the interval between herbicide treatment of unwanted vegetation and direct drilling of spring barley was shortest, e.g. 2-3 days from herbicide treatment to spring planting. Disease damage was least when intervals between weed treatment and spring planting were longest, e.g. weed treatment in the previous autumn with crops sown in spring. Recent studies have yielded similar results; disease damage to spring seedlings of onion [55] and corn [56] was minimised when the time interval between GBH application to cover crops of winter cereals and planting of the spring crop was maximised. For example, when the time interval between GBH application and onion planting increased from 3 days to 19 and 27 days, the total area of onions affected by stunting due to infection by Rhizoctonia solani decreased by $54-63 \%$ [55]. Taken together, the above findings are inconsistent with commercial recommendations that sowing may proceed as little as $4-72 \mathrm{~h}$ after herbicide treatment (see "The fate of glyphosate in the soil").

\section{Repeated exposure and shifting population dynamics}

It is important to consider the longer-term implications of the findings presented in "Stimulation of phytopathogenic microorganisms" above by considering whether chronic/repeated exposure to GBHs may drive a selective shift in the rhizosphere microbial community, favouring glyphosate-tolerant microbial species over sensitive ones. This selective process could have potentially deleterious consequences for crop health where phytopathogenic species are encouraged. Although extensive research has been done on short-term microbial responses to GBHs (see "Stimulation of phytopathogenic microorganisms"), only a small number of studies have sought to observe longer-term (multi-year) effects [37, 57-59]. These studies and their findings are summarised as follows.

Collated root colonisation data from Kremer and Means [37] indicate that an annual programme of GBH treatments may have encouraged gradual expansion of the glyphosate-tolerant Fusarium spp. population in their experimental plots over the 10 years of their field study. Relatively low levels of soybean root colonisation were detected during years 1-4 of the study (e.g. 20-40 Fusarium colonies per $100 \mathrm{~cm}$ section of soybean root) compared with marked increases during years $5-10$ of the study (100-120 Fusarium colonies per $100 \mathrm{~cm}$ section of soybean root).

Zabaloy et al. [59] compared the respiration rate (Fmax) of microorganisms present in field soils with differing management histories. A 'pristine' grassland soil with no previous exposure to glyphosate and two 'no-till' agricultural soils with 15 and 11 years of exposure to GBHs, respectively, were treated with glyphosate (Nidera, 95\% technical grade) in a microcosm experiment. The soil samples had been pre-incubated for $24 \mathrm{~h}$ to reduce the levels of endogenous nutrient and afterwards for a further week for reasons not given. The 'pristine' soil exhibited a significant elevation in ( F max) (determined by measurement of oxygen use) in response to glyphosate treatment, compared with untreated controls and with the two 'no-till' soils, which showed either unaffected or decreased Fmax following glyphosate application. The authors attributed the elevated microbial respiration rate in the 'pristine' soil to a stress response, inferring that a larger proportion of the soil's microbiota may have been vulnerable to glyphosate toxicity. They suggested that increased microbial respiration may occur under glyphosate-induced stress due to the quantity of ATP diverted towards accumulation of shikimate and hydroxybenzoic acids following EPSPS disruption [59, 60]. It is questionable, however, whether such an accumulation (which is due to an inhibition and not an increased synthesis) would result in such a diversion of ATP. An alternative explanation could be that the increased respiration results from an uncoupling of oxidative phosphorylation by glyphosate. ${ }^{1}$ Nicolas et al. [61] provided evidence for this in fungal mitochondria. In Aspergillus nidulans, germination, growth and development were all inhibited by very low concentrations of Roundup, yet mitochondrial respiration was increased.

While it is not possible to determine (based on the available evidence) whether the increase in microbial respiration observed by Zabaloy et al. [59] had indeed occurred in response to glyphosate stress, the contrast in responses between the two samples does suggest that different management histories (i.e. chronic GBH exposure vs. no GBH exposure) had resulted in microbial communities that were either structurally or functionally different from one another, and which responded to glyphosate treatment in different ways.

Allegrini et al. [58] subsequently applied a 'pollutioninduced community tolerance' (PICT) approach to assess whether chronic glyphosate exposure might exert selective pressure on microbial community structure and result in increased microbial GBH tolerance. The authors hypothesised that microbial tolerance to GBHs would be higher in soils with a multi-year history of GHB exposure

\footnotetext{
1 That glyphosate can uncouple oxidative phosphorylation has been known for some time [62] and was quoted in the National Pesticides Information Centre Fact Sheet [7]. Recently, Swanson et al. [63] gave a detailed account of how glyphosate affects mammalian mitochondrial function, including by uncoupling.
} 
than in soils that never encountered the herbicides. However, in contrast to the conclusions of Zabaloy et al. [59], the PICT assays found that microbial response to a $\mathrm{GBH}$ treatment was unrelated to a previous history of herbicide exposure (the microbial respiration rate was not significantly different between soils) and the study as a whole identified no clear evidence for localised microbial adaptation to GBHs.

Finally, a study conducted by [57] compared bacterial community composition in the rhizospheres of two crops. Corn [Zea mays; cultivar DKC62-54 (VT3)] and soybeans (Glycine max; cultivar OX 20-8 RR) were grown in a soil with no previous history of GBH exposure, within rhizoboxes. The GBH (Roundup Powermax, at recommended field rates) was applied to both crops (once prior to sowing, and twice more when plants reached V4 and V7 growth stages, respectively) during each of four 58-day cropping cycles. At the end of the fourth cropping cycle, the authors used next-generation barcoded sequencing to identify specific bacterial taxa shifts occurring in the rhizospheres of GBH-treated plants, compared with those of untreated control plants. For both corn and soybean crops, the study revealed subtle alterations to microbial community composition in response to $\mathrm{GBH}$ treatment. The authors observed an increase in the relative abundance of sequences associated with members of the phylum Proteobacteria $(p=0.096)$ in rhizospheres of both corn and soybean (e.g. from an average of $22.9 \pm 1.5 \%$ in control samples to $25.9 \pm 0.9 \%$ in the rhizosphere of GBH-treated corn). The increase in relative abundance of the phylum Proteobacteria was driven by increases in sequences from the family Xanthomonadaceae; the authors inferred that these may have been enriched by GBH exposure. In contrast, the relative abundance of the phylum Acidobacteria showed a decrease in response to $\mathrm{GBH}$ treatment $(p=0.083)$ in rhizospheres of both crops (e.g. from an average of $21.5 \pm 1.1 \%$ in control samples to $18.7 \pm 0.8 \%$ in the rhizosphere of GBH-treated corn). Since some members of the Acidobacteria are thought to be important contributors to biogeochemical processes within the rhizosphere, the authors suggested that a consistent decrease in their abundance over time could lead to changes in the nutrient status of the rhizosphere and could impact on the health and productivity of crops.

\section{Three potential factors underlying stimulation of phytopathogens}

Nutritional stimulation Numerous microbial species are able to metabolise glyphosate as a direct source of nutrition [23, 64-70] Some rhizosphere-inhabiting Fusarium spp., for example, have been shown to metabolise glyphosate in plant root exudates as a source of phosphorus $(\mathrm{P})$, carbon (C) and energy [68]. In addition, herbicide-induced changes to the composition and/or the quantity of treated plant root exudates may further enhance the nutritional richness of the rhizosphere for microorganisms poised to metabolise these products. Following treatment of two soybean cultivars (GR; 'Pioneer 94B01', and GS; 'Williams 82') with a GBH (Roundup Ultra; Monsanto, St Louis, $\mathrm{MO}$ ) at a rate of $0.84 \mathrm{~kg}$ a.e. $\mathrm{ha}^{-1}$, Kremer et al. [23] found that glyphosate residues were exuded from roots of both cultivars in steadily increasing concentrations from 2 up to 12 days after herbicide treatment. From 12 to 16 days after treatment, glyphosate exudation continued at a nearconstant rate from the living roots of the GR cultivar, but diminished in the GS 'W82' cultivar, apparently slowed by the death of the plants. In addition, it was observed that root exudates of treated soybeans (both GS and GR) contained high concentrations of free amino acids and soluble carbohydrates, compared with the exudates of untreated controls. These products are rich nutrient sources for bacterial and fungal phytopathogens, as well as insect pests. When cultured directly in the root exudates of treated plants, select Fusarium spp. strains (strains 301 and 304) developed significantly higher microbial biomass compared with controls [23]. Likewise, Liu et al. [71] found that germination and growth of Pythium ultimum germ tubes was significantly enhanced when isolates of the pathogen were cultured in root exudates of Rounduptreated bean seedlings, compared with those of untreated controls.

Neither of the two studies [23, 71] was able to distinguish the stimulatory effects of exuded carbohydrates and amino acids from those of exuded glyphosate. Nevertheless, it appears that the combined release of residual glyphosate, along with elevated quantities of soluble metabolic products from the roots of GBH-treated plants, may have been of direct benefit to certain opportunistic phytopathogens present in the rhizosphere. This is consistent with Chaboussou's theory of trophobiosis [72] and references therein (see below), which states that phytopathogenic microorganisms proliferate where these less complex metabolic products (i.e. free amino acids and reducing sugars such as glucose as opposed to complex proteins and carbohydrates, respectively) are available in excess.

Impairment of the physiological defence mechanisms of crops Since key components of the physiological disease resistance of crops, e.g. the production of various defensive metabolites and the protective lignification of cell walls, are dependent on the products of the shikimic acid pathway, exposure to glyphosate (even at sub-lethal doses) has the potential to impair innate disease resistance in plants $[46,71,73]$. 
Inhibition of EPSPS restricts the biosynthesis of key phenolic defence products such as antibiotic compounds, pathogen-induced anti-microbial phytoalexins, and cinnamic acid-derived lignin for structural cell wall enhancement at infection sites, [6, 42]. The inhibition of EPSPS by GBHs has been shown to inhibit the formation of defensive metabolites to the extent that the innate resistance of a crop to a pathogen is lost, or weakened $[42,47,54,74-76]$.

Early laboratory studies demonstrated that sub-lethal doses of glyphosate prevented accumulation of the defensive phytoalexin glyceollin in GS soybean [Glycine max (L.) Merr. 'Harosoy 63'], resulting in increased susceptibility of the previously resistant soybean cultivar to infection by two pathogenic fungi, Phytophthora megasperma f. sp. glycines and Pseudomonas syringae pv. glycinea [73]. Sub-lethal doses of glyphosate applied to seedlings of two Fusarium oxysporum-resistant GS tomato cultivars were shown to severely undermine the innate resistance of tomato plants to the pathogen, resulting in severe $F$. oxysporum colonisation of tomato root tissues [76]. Sharon et al. [46] reported a substantial (fivefold) decrease in the concentration of Alternaria cassia conidia required to kill seedlings of Senna obtusifolia L. when a sub-lethal dose of glyphosate was applied in combination with the fungal inoculum, and Liu et al. [71] reported that when bean seedlings (Phaseolus vulgaris L.) were treated with Roundup ${ }^{\circledR} 2$ days before inoculation with mycelial suspensions of Pythium ultimum, defensive pathogeninduced lignification in plant roots was significantly suppressed compared with controls, rendering treated plants significantly more vulnerable to Pythium ultimum damage.

The collective results of these studies indicate clearly that exposure to GBHs, even at sub-lethal doses such as may occur by accidental drift, can result in significant inhibition of GS crops' physiological defence mechanisms and thereby increased disease damage of crops.

Both Cerdeira and Duke [77] and Duke et al. [6] suggested that since GR crops express a glyphosate-tolerant form of EPSPS, herbicide-mediated impairment of plant defences via disruption to phenolic metabolism is unlikely to occur in Roundup Ready ${ }^{\circledR}$ cultivars. This reasoning is logical if it is assumed that the GR EPSPS enzyme functions equivalently to the wild-type EPSPS enzyme in the absence of herbicide, and is equally efficacious in supporting disease-resistance mechanisms when exposed to herbicide. A few studies have indicated, however, that some GR cultivars are also vulnerable to inhibition of physiological defences following GBH application at sub-lethal doses $[47,78,79]$.

Larson et al. [78] demonstrated herbicide-induced loss of resistance in the previously Rhizoctonia root rot-resistant GR sugar beet cultivar, 'B4RR'. GBH-treated (Roundup WeatherMAX; Monsanto Co., St Louis, MO, at a rate of $0.84 \mathrm{~kg}$ a.e. $\mathrm{ha}^{-1}$ ) sugar beet suffered significantly enhanced disease damage when inoculated with an isolate of Rhizoctonia solani to which it would ordinarily be resistant, compared with untreated control plants. The authors interpreted the observed loss of resistance to be a 'plant-mediated' phenomenon, since in vitro $R$. solani growth assays showed no significant alterations in the growth rate of fungal isolates when cultured with the herbicide at equivalent concentrations, compared with controls. Larson et al. [78] took repeated measurements of shikimic acid accumulation (a reliable marker for EPSPS disruption) in GR sugar beet tissues at regular time intervals $(0,3,7$ and 14 days following glyphosate application). Seedlings showed significantly higher shikimate accumulation in all tissue types, except roots, and at all sampling time points in glyphosate-treated plants compared with controls. The authors proposed that the observed disruption of sugar beet EPSPS following glyphosate exposure (indicated by the increased levels of shikimate) might have been sufficient to disrupt physiological defence mechanisms, increasing the susceptibility of the plant to infection by the $R$. solani pathogen.

The findings of Larson et al. [78] are consistent with those of Sanogo et al. [47], who found that when two previously Fusarium solani-resistant GR soybean cultivars (Asgrow 3701, and Asgrow HIG3071) were inoculated with two isolates of F. solani (isolates 'Monticello', and 'Scott'), glyphosate treatments (Roundup Ultra, at $0.84 \mathrm{~kg}$ a.e. $\mathrm{ha}^{-1}$ ) resulted in statistically significant increases in the severity of sudden death syndrome (measured as \% Fusarium damage to leaves and roots) occurring in plants of both cultivars, as well as significantly increased isolation frequency of $F$. solani sp. glycines from plant roots. Conidial germination, mycelial growth and sporulation of F. solani isolates ('Monticello', and 'Scott') were reduced when glyphosate was added to the culture medium, compared with controls [47], indicating that the enhanced disease development observed in planta had occurred due to herbicidal suppression of plant immunity, rather than to direct stimulation of the pathogens.

Finally, a greenhouse study by Zobiole et al. [79] found that when GR soybean plants (Glycine max L. Merr. Cultivar BRS-242 GR) were treated with glyphosate (isopropylamine salt), soybean lignin production decreased markedly with increasing glyphosate application rates. The total lignin content of plants decreased from $\sim 0.50 \mathrm{~g}$ plant- 1 in untreated soybeans, to $\sim 0.14$ g plant- 1 in soybeans treated with glyphosate at a rate of 1800 g a.e. ha ${ }^{-1}$. The authors also reported a significant decrease in the total amino acid content of treated soybean plants relative to controls, but we question the units presented with 
this data. Since lignin biosynthesis is dependent on phenylalanine, a key amino acid product of the shikimate pathway, the authors proposed that the observed declines in lignin production might have been due to herbicideinduced EPSPS disruption, even in their GR soybeans. Possibly, declines in soybean photosynthetic rate and chlorophyll content with increasing glyphosate application rates may also have contributed to the observed suppression of lignin biosynthesis. The authors do not comment on any reduction in protein synthesis [79].

Suppression of pathogen antagonists A third factor may be the GBHs suppression of beneficial rhizosphere-dwelling microbial species which antagonise the pathogens. As mentioned above, certain species express glyphosatesensitive forms of microbial EPSPS and suffer metabolic disruption when exposed to GBHs.

Several studies have reported declines in rhizosphereinhabiting populations of fluorescent Pseudomonas spp. (ubiquitous soil bacteria capable of synthesising various defensive metabolites and antibiotics, and enhancing nutrient availability), following $\mathrm{GBH}$ applications to GR soybeans. For example, during the course of their long-term field study, Kremer and Means [37] reported significant decreases in rhizosphere-populations of fluorescent Pseudomonas spp. following GBH treatment of GR soybeans. Pseudomonas decline correlated with the observed increases in root colonisation by Fusarium spp., and bioassays of single cultures confirmed that $~ 85 \%$ of the identified Pseudomonas spp. were potentially antagonistic towards Fusarium spp. Correspondingly, the aforementioned study by Zobiole et al. [36] reported a decline in rhizosphere-inhabiting fluorescent pseudomonads following application of Roundup WeatherMAX to soybeans (GR1; BRS242 and GR2; AG3539).

A detailed analysis by Aristilde et al. [35] illustrated the significant variation that exists between rhizospheredwelling Pseudomonas species and even between strains, in degrees of sensitivity to glyphosate at varying concentrations. In sensitive species, glyphosate was shown to significantly inhibit the biosynthesis of the essential amino acids phenylalanine, tyrosine and tryptophan via disruption to bacterial EPSPS [35].

Herbicide-induced suppression of sensitive Pseudomonas spp. has the potential to impact detrimentally on the disease resistance of crops by: (i) reducing the availability of Pseudomonas spp.-derived secondary metabolites which are contributors towards plant disease defences and (ii) reducing the competitive antagonism provided by Pseudomonas spp. towards phytopathogenic species such as Fusarium, allowing these to proliferate. Fernandez et al. [53, 80] also detected GBH-induced alterations to competitive relationships between fungal species, since weed control with GBHs appeared to have a stimulatory effect on Fusarium spp. (causal agents of Fusarium head blight, root and crown rot, and sudden death syndrome) while suppressing Cochliobolus sativus (causal agent of common root rot, a common disease of wheat and barley). The authors inferred that the observed suppression of Cochliobolus sativus populations might have contributed to the relative proliferation of Fusarium spp. populations, due to a reduction in competitive antagonism.

\section{Pathway II: impacts on plant mineral nutrition}

Balanced nutrition is essential for the regulation of plant metabolic processes supporting physiological disease resistance [42,81], and crops are more susceptible to disease when their nutritional balance is not optimal, i.e. when nutrients are not available in their optimal ratios [72, 81]. GBHs have been shown to interfere with the uptake, in-plant translocation and utilisation of essential elements (e.g. metals) within crops, including those exposed to sub-lethal doses, and even to concentration levels associated with herbicide drift [24, 82-84]. Researchers have raised concerns that disruptions of this kind may compromise the innate disease resistance, elevating the risk of disease in cropping systems where GBHs are used for weed control [42]. The potential for such a relationship warrants closer scrutiny. Accordingly, this section outlines the limited published studies that examine the effects of GBHs on crop nutrition, first in the context of GS crops and then with regard to GR crops.

\section{Nutritional disruption in GS crops}

Eker et al. [83] demonstrated that root uptake of both radiolabelled manganese $\left({ }^{54} \mathrm{Mn}\right)$ and iron $\left({ }^{59} \mathrm{Fe}\right)$ was markedly reduced ( $\sim 25$ and $\sim 75 \%$, respectively) compared with controls, following treatment of sunflower seedlings with sub-lethal concentrations of a GBH (Roundup Ultra, Monsanto co., at 6.0\% label-recommended dosage). Additionally, herbicide treatments resulted in near-complete inhibition of root-shoot translocation of ${ }^{59} \mathrm{Fe}$ and ${ }^{56} \mathrm{Mn}$, at 12 and $24 \mathrm{~h}$ post-treatment, respectively. Both uptake and translocation of radiolabelled zinc $(65 \mathrm{Zn})$ also decreased, although not statistically significantly. Cakmak et al. [82] raised seedlings of GS soybean in soils amended with a standard complement of macro- and micronutrients. At the V4, V6, and early R1 growth stages, seedlings were treated with a GBH (Roundup Ultra, Monsanto Ltd, Turkey) at sub-lethal concentrations representing between 0.3 and $1.2 \%$ of the label-recommended dose. At soybean maturation, concentrations of calcium (Ca), magnesium ( $\mathrm{Mg}$ ), $\mathrm{Mn}$ and $\mathrm{Fe}$ were each reduced in the beans produced by herbicidetreated plants, in a dose-dependent manner. Reductions 
in nutrient concentrations were most extreme (e.g. Ca, $\mathrm{Mg}, \mathrm{Mn}$ and Fe by $\sim 26, \sim 13, \sim 45$ and $\sim 49 \%$, respectively) when plants were exposed to the highest herbicide dosage ( $1.2 \%$ of the recommended field rate). Finally, the findings of Ozturk et al. [84] indicate that high sensitivity of the enzyme Fe-reductase to very low concentrations of $\mathrm{GBH}$ may play a role in glyphosate-mediated impairment of $\mathrm{Fe}^{-}$uptake. They [84] investigated the effects of drift concentrations of a GBH (Roundup Ultra, Monsanto Ltd, Turkey, at concentrations corresponding to 1,3 and $6 \%$ of the recommended dose for weed control) on the activity of ferric $\left(\mathrm{Fe}^{3+}\right)$ reductase in root tissues of Fe-deficient sunflower seedlings. Glyphosate exposure inhibited root Fe-reductase activity in a dose-dependent manner. At the highest dose $(1.89 \mathrm{mM}$, corresponding to $6 \%$ of recommended herbicidal dose), Fe-reductase activity was inhibited by $50 \%$ at $6 \mathrm{~h}$ after treatment and by almost $100 \%$ at $24 \mathrm{~h}$ after treatment.

The above results suggest a significant influence of GBHs on the uptake of some micronutrients, their rootto-shoot translocation and, in the case of the study by Cakmak et al. [82], on their accumulation in mature GS soybeans. Importantly, these findings demonstrate the potential for nutritional disruption of this kind to occur following exposure of GS plants to a small fraction of the recommended herbicidal dosage, such as may occur by accident due to spray drift or root contact with herbicide residues in soil. Mechanisms underlying the observed antagonism between GBHs and crop nutrition were not conclusively identified by these studies. It has been suggested, however, that the observed declines in uptake, as well as in-plant transport of micronutrients, might be related to the ability of glyphosate to form poorly soluble glyphosate-metal chelates within plant tissues and/or within the rhizosphere [82, 83]. Ozturk et al. [84] inferred that the formation of glyphosate-Fe complexes might have restricted the availability of $\mathrm{Fe}(\mathrm{III})$, crucial for the maintenance of Fe-reductase enzyme activity, in plant cells.

Since glyphosate binds with metal ions (see "Metalbinding properties of glyphosate"), the formation of glyphosate-metal chelates in spray solutions has been shown to limit penetration and translocation of glyphosate within plants [85-87]. In turn, it is possible that formation of glyphosate-metal chelates where glyphosate accumulates in root tissues [21] and/or in the rhizosphere [23] may restrict the availability of nutrient metals for in-plant transport and use [24, 82, 83]. Alternatively or in addition, it is possible that the nutritional disruption observed may have occurred due to low-level herbicide toxicity from the accumulation of glyphosate (and/or its primary breakdown product, AMPA) in crop roots, where partial impairment of GS EPSPS could have impaired the physiological processes associated with nutrient acquisition [6].

\section{Glyphosate-nutrient antagonism in GR crops}

There are conflicting conclusions in the literature about whether GBHs might impact adversely upon mineral nutrition in GR crops. A detailed review by Duke et al. [6] acknowledges that since glyphosate alters almost every physiological and biochemical process during the course of its herbicidal action in sensitive plants, it follows that the nutrition of GS crops is likely to be compromised following application of a $\mathrm{GBH}$ at recommended concentrations. In contrast, they also propose that GBHs should not be expected to disrupt nutritional physiology in resistant GR cultivars, due to the engineered resistance of these crops to EPSPS disruption. Indeed, several studies have detected no deleterious impacts of GBHs on GR soybean nutrition [88-91]. Among these, Duke et al. [91] detected minimal and inconsistent effects of a GBH (Roundup WeatherMAX, applied at $0.87 \mathrm{~kg}$ a.e $\mathrm{ha}^{-1}$ ) on foliar and seed content of $\mathrm{Al}, \mathrm{As}, \mathrm{Ba}, \mathrm{Cd}, \mathrm{Co}, \mathrm{Cr}, \mathrm{Cs}, \mathrm{Fe}$, $\mathrm{Cu}, \mathrm{Fe}, \mathrm{Ga}, \mathrm{K}, \mathrm{Li}, \mathrm{Mg}, \mathrm{Mn}, \mathrm{Ni}, \mathrm{Pb}, \mathrm{Rb}, \mathrm{Se}, \mathrm{Sr}, \mathrm{TL}, \mathrm{U}, \mathrm{V}$ and $\mathrm{Zn}$, in GR soybeans (cultivar USG Allen GR). There were also no effects of $\mathrm{GBH}$ treatment on soybean grain yield [90].

However, a small number of studies have reported significant disruptions to GR crop nutrition, as well as declines in plant biomass production, chlorophyll content and photosynthetic rate, associated with GBH treatments at the recommended herbicidal dosage to GR crops [20, 79, 92-96]. These studies and their main findings are summarised as follows.

Serra et al. [93] found that accumulation of Fe, $\mathrm{Zn}, \mathrm{Mn}$ and $\mathrm{Cu}$ was significantly reduced in GR soybeans (cv. P98R31 RR) at the V8-growth stage, when these were treated with glyphosate (formulation unspecified) at

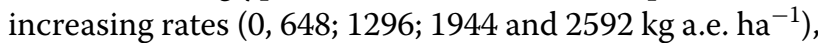
compared with controls. Zobiole et al. [94] grew GR soybeans of three cultivars (BRS242 GR, BRS245 GR and BRS247 GR, representing early, medium and late maturity groups, respectively) in two soil types (a Typic Hapludox, and a Rhodic Ferralsol). At the V4 and V4 + V5 growth stages, soybeans were treated with either one $\left(1200\right.$ g a.e. $\left.\mathrm{ha}^{-1}\right)$ or two $\left(600+600 \mathrm{~g}\right.$ a.e. $\left.\mathrm{ha}^{-1}\right)$ applications of glyphosate (as the isopropylamine salt), respectively. When measured at the R1 growth stage, leaf concentrations of $\mathrm{Mn}, \mathrm{Fe}, \mathrm{Cu}$ and boron (B) were each significantly reduced in herbicide-treated plants of the early and medium maturity cultivars. Leaf concentrations of $\mathrm{Zn}$ were also significantly reduced in herbicidetreated plants of the early maturity cultivar, as were the macronutrients phosphorus (P), potassium (K), $\mathrm{Ca}, \mathrm{Mg}$ and sulphur (S). Only $\mathrm{P}$ and $\mathrm{Mn}$ concentrations were 
reduced significantly in the late maturity cultivar, compared with controls. The authors inferred that the later maturing cultivar might have benefitted from a longer period of recovery post-treatment, compared with the early and medium varieties that showed greater impacts. In a second greenhouse experiment, Zobiole et al. [95] raised GR soybeans of two cultivars (BRS242 RR1 and AG3539 RR2) on a Mexico silt loam soil. At the V2, V4 or V6 growth stages, soybeans were treated with Roundup WeatherMAX $^{\circledR}$ (Monsanto co.) at 800,1200 or $2400 \mathrm{~g}$ a.e. $\mathrm{ha}^{-1}$. In herbicide-treated soybeans of both cultivars, leaf concentrations of several micronutrients $(\mathrm{Zn}, \mathrm{Mn}$, $\mathrm{Fe}, \mathrm{Cu}, \mathrm{B})$ and macronutrients $(\mathrm{N}, \mathrm{P}, \mathrm{Mg}, \mathrm{K}, \mathrm{Ca}, \mathrm{Mg}, \mathrm{S}$ ) were markedly reduced compared with controls at the R1 growth stage. In both experiments, and across all cultivars and soil types, reductions in leaf nutrient concentrations were accompanied by significant declines in the chlorophyll content of soybean leaves, as well as significant declines in the production of root and shoot biomass $[94,95]$. Photosynthetic rate was also reduced in herbicide-treated plants of the medium and late maturity cultivar [97]. On the one hand, it is possible that accumulation of glyphosate (and/or its phytotoxic metabolite AMPA) in soybean sink tissues may be the direct cause of the observed chlorotic symptoms, since both glyphosate and AMPA have been shown to damage chlorophyll [89, 98-100]. For example, Gomes et al. [100] proposed that due to their chemical similarity, AMPA may compete with the essential amino acid glycine and thereby interfere with/inhibit its role in the biosynthesis of chlorophyll. Reduced photosynthetic efficiency may, in turn, reduce the energy allocated to root growth and restrict the capacity of the plant for nutrient uptake. Alternatively, it is possible that reduced leaf chlorophyll concentrations may have occurred as a secondary impact of the physiological inactivation of essential micronutrients (e.g. $\mathrm{Mn}, \mathrm{Mg}$ ) due to glyphosate-metal complexation.

Other authors have found GBH-induced reduction in nutrient uptake by GR crops to be strongly dependent on selected culture conditions [92, 96], as well as on differences between the herbicide formulations tested [20]. For example, when GR soybeans (cv. Valiosa RR) were supplied with sufficient Mn $(0.5 \mu \mathrm{M})$ in hydroponic growth chambers, GBH treatments (Roundup ${ }^{\circledR}$ UltraMax $^{\circledR}$ ) at the label-recommended dose reduced shoot Mn concentrations by $\sim 50 \%$, compared with controls. Root growth and elongation was also significantly reduced $(\sim 30 \%)$ in GBH-treated soybeans. No comparable responses occurred, however, in GBH-treated soybeans cultured with low Mn supply $(0.1 \mu \mathrm{M})$. Likewise, leaf $\mathrm{Zn}$ concentrations were significantly reduced by treatments with Roundup $^{\circledR}$ Ultramax when soybeans (cv. Valiosa RR) were cultured in a sandy, acidic arenosol, but not on a calcareous loess sub-soil of luvisol [96]. Petter et al. [92] found that soil water conditions can strongly influence the degree to which nutrient accumulation is impaired by GBH exposure in GR soybeans. Under adequate moisture conditions, GBH applications (Roundup Ready; at doses of $1080 \mathrm{~g}$ and $1800 \mathrm{~g}$ a.e. $\mathrm{ha}^{-1}$ ) significantly reduced the accumulation of the macronutrients $\mathrm{N}, \mathrm{P}, \mathrm{K}, \mathrm{Ca}$ and $\mathrm{Mg}$ and of micronutrients $\mathrm{B}, \mathrm{Mn}, \mathrm{Zn}$ and $\mathrm{Fe}$ in all three GR soybean cultivars tested (P98Y12RR, M9144RR and M9056RR) in a dose-dependent fashion. This was with the exception of one cultivar (M9144RR), in which $\mathrm{N}$-accumulation was reduced, but not statistically significantly. In several, but not all cases, GBH-induced reduction in nutrient accumulation was significantly greater when plants were grown under conditions of soil water deficit [92].

Finally, a report by Zobiole et al. [101] found that GBHinduced inhibition of GR soybean metal uptake could be reversed by soil supplementation with a mixture of amino acids, including glycine. It is conventionally understood that GR crops should not be deficient in amino acids following treatment with a $\mathrm{GBH}$, due to their resistance to glyphosate's primary mode of action on plant EPSPS. However, the finding that addition of glycine reverses the effect of GBHs on GR crop mineral nutrition [101] suggests that GBHs may interfere with either the synthesis or function of glycine in GR soybeans. As suggested above with regard to chlorophyll synthesis [100], it seems possible that glyphosate and/or AMPA may be acting as a competitive analogue of glycine, potentially outcompeting the amino acid in biological sites and pathways. This could have knock-on effects for numerous plant physiological functions, including nutrient uptake.

Taken together, the above findings indicate that GBHs do have the potential to interfere with mineral nutrition in GR cultivars under some conditions, despite the engineered resistance of these cultivars to glyphosate's intended mode of action on plant EPSPS. We repeat that herbicidal impacts appear to be strongly influenced by growth conditions such as soil properties. Interestingly, to our knowledge, the majority of papers which conclude that GBHs do interfere with GR crop nutrition used commercially formulated GBH products in their experimental trials (see above), whereas the majority of papers that do not identify any interference used pure glyphosate in their trials. The exceptions are two recent studies by Duke et al. [90, 91], both of which applied a commercial $\mathrm{GBH}$, and both of which concluded that there was no significant effect of GBH treatment on the mineral content of GR soybeans. It is noteworthy that Duke et al. $[6,89$, 90] pre-treated their experimental soils with other herbicides ( $S$-metolachlor, pendimethalin and paraquat) which could have had unidentified influence on their findings, 
for instance by pre-empting further effects from GBHs. We propose that the contrasting conclusions put forward may be explained, at least in part, by the different herbicides used, since formulated products are likely to act differently in plants and soils to the active ingredient alone.

None of the mechanisms behind the observed interferences have been conclusively identified, although several authors cited glyphosate-metal chelation as a potential underlying factor [94-96]. Other possible factors include sub-lethal toxicity and impairment of soybean root physiology following accumulation of residual glyphosate and/ or AMPA in root tissues $[99,100]$, and herbicide-induced disruption to rhizosphere-dwelling organisms associated with nutrient acquisition (see "Pathway I: disruptions to rhizosphere microbial ecology" section). Further investigation is clearly necessary to determine the relative contributions of the several, likely interrelated mechanisms which underlie the observed interference and their degree of expression in relevant agricultural contexts.

Nutrient restrictions of the kind observed in the above studies are likely to interfere with the physiological disease resistance of crops. If GBHs do indeed disrupt the nutrient status of crops significantly, then their diseaseinducing impacts could be explained, at least in part, by Chaboussou's [72] theory of trophobiosis. This, originally published in French in 1985, before the use of GBHs and GR crops, has been a widespread theory. Chaboussou showed, citing reviewed papers as well as his own experimental work, that disease-causing organisms are stimulated to proliferate when concentrations of free amino acids and reducing sugars are elevated in plant cells. These products are rich nutrient sources for bacterial and fungal phytopathogens, as well as insect pests. Any, even temporary, disruption of macromolecular synthesis results in elevated cellular concentrations of soluble amino acids and sugars, thus providing an environment where disease organisms are encouraged. Since various metal ions are involved in macromolecular (especially protein) synthesis, changes in their relative proportions are likely to be disruptive. This idea of trophobiosis would provide an overarching view of how nutrition affects disease, and hence account for one aspect of how GBHs may cause damage through their effects on the availability of nutrient metal ions.

\section{Conclusions}

Cumulatively, the findings from the peer-reviewed literature presented in this review article counter the assumption that GBHs are innocuous when applied according to the manufacturers' recommendations. The review sheds light on the extremely complex network of influences that glyphosate-based weed control techniques may have upon crops (of both GS and GR varieties) and upon their interactions with microbial phytopathogens.

It is important to recall, however, that considerable variability exists within these research findings, making it very difficult to reach generalised conclusions. We identified several factors that underlie this variability between different experimental studies including: (i) the properties of experimental soils and their mineral contents may differ greatly; (ii) the crop species and cultivars are often different; and (iii) commercial GBH formulations are formulated differently, frequently with unspecified ingredients (e.g. adjuvants) whose impacts are difficult to distinguish from those of the active ingredient. Numerous reports, including several of those cited above, show that formulated GBHs are orders of magnitude more active than glyphosate or its salts.

Nevertheless, one can draw a number of clear conclusions from the research collated here: (i) GBHs are shown to strongly undermine the innate physiological defences of GS crops by impairment of the shikimic acid pathway and by other biochemical effects; these render crops significantly weaker and more vulnerable to pathogenic attack; (ii) impairment of physiological disease defences has also been shown to occur in some GR cultivars, despite their engineered resistance to glyphosate's primary mode of action; (iii) GBHs are shown to interfere with local microbial ecology upon their release to the rhizosphere. GBHs have the potential to promote phytopathogenic microbial species, through multiple inter-linked mechanisms (which include direct nutritional stimulation, as well as suppression of antagonists), enhancing their virulence and their damage to crops; and finally, (iv) the as yet incompletely understood influences of GBHs on the uptake and utilisation of nutrient metals by crops has the potential to further impair disease resistance. Importantly, the above deleterious impacts may occur in synergy with each other, and so compound and intensify each other's damaging consequences.

The above conclusions are sufficient to conclude that glyphosate and its related products are not at all innocuous, in either GS or GR cropping systems. Rather, it is clear that the action of GBHs is not limited to the disruption of EPSPS in target plants, but can be far reaching. While we cannot conclude that the above impacts will occur in every case (we repeat that environmental conditions, crop cultivars and individual farming practices will strongly influence outcomes), and we can conclude that GBHs have the capacity to cause significant harm or impairment to the crops that they are employed to protect. Such a situation calls for urgent reconsideration of the wisdom of their widespread and intensive application in agriculture. These conclusions add to and complement 
the already substantial literature reporting the damaging effects of GBHs on animal life $[4,102]$.

Overall, we believe that the effects of GBHs are potentially wide ranging and are fundamentally linked to herbicide action. Our conclusions highlight the difficulty in developing a herbicide that is effective yet innocuous in relation to all non-target species and wider ecosystems. There exists a great need for collaboration between growers, regulators and industry to develop products, practices and policies that minimise the use of herbicides as far as possible and maximise their effectiveness when they are used, while facilitating optimised food production and security.

\section{Authors' contributions}

DAM carried out most of the literature searches, initially for her student dissertation and then independently, and wrote much of the review. MCG and UEL acted initially as supervisors and then as co-researchers. All authors read and approved the final manuscript.

\section{Author details}

${ }^{1}$ Formerly School of Geosciences, University of Edinburgh, Edinburgh, Scotland, UK. ${ }^{2}$ Ormiston Hall, Formerly Centre for Human Ecology and Department of Zoology, University of Edinburgh, EH35 5NJ Edinburgh, Scotland, UK. ${ }^{3}$ School of GeoSciences, Crew Building, The King's Buildings, University of Edinburgh, Alexander Crum Brown Road, EH9 3JF Edinburgh, Scotland, UK.

\section{Acknowledgements}

This work was developed from a dissertation presented as part fulfillment of the degree of BSc in environmental science (ecology) by D. A. M. We thank The Sheepdrove Trust for their support to D. A. M. We also thank Dr. lan W. Oliver for many helpful comments on the manuscript and the reviewers for their care and suggestions, which have improved the paper.

\section{Competing interests}

The authors declare that they have no competing interests.

\section{Availability of data and materials}

Not applicable.

\section{Consent for publication}

Not applicable.

\section{Ethics approval and consent to participate}

Not applicable.

\section{Funding}

The authors acknowledge with thanks a small personal grant to DAM from the Sheepdrove Trust, to enable her to proceed with the work.

\section{Publisher's Note}

Springer Nature remains neutral with regard to jurisdictional claims in published maps and institutional affiliations.

Received: 6 September 2017 Accepted: 6 January 2018

Published online: 16 January 2018

\section{References}

1. Duke SO, Powles SB (2008) Glyphosate: a once-in-a-century herbicide. Pest Manag Sci 64:319-325

2. Benbrook C (2012) Impacts of genetically engineered crops on pesticide use in the US - the first sixteen years. Environmental Sciences Europe 24:24
3. Benbrook CM (2016) Trends in glyphosate herbicide use in the United States and globally. Environ Sci Eur 28:1

4. Myers JP, Antoniou MN, Blumberg B, Carroll L, Colborn T, Everett LG, Hansen M, Landrigan PJ, Lanphear BP, Mesnage R, Vandenberg LN, Vom Saal FS, Welshons WV, Benbrook CM (2016) Concerns over use of glyphosate-based herbicides and risks associated with exposures: a consensus statement. Environ Health 15:19

5. Monsanto, The agronomic benefits of glyphosate in Europe-Benefits of glyphosate per market use. Monsanto Int sàrl Monsanto Eur, 2010, 1-82

6. Duke SO, Lydon J, Koskinen WC, Moorman TB, Chaney RL, Hammerschmidt R (2012) Glyphosate effects on plant mineral nutrition, crop rhizosphere microbiota, and plant disease in glyphosate-resistant crops. J Agric Food Chem 60:10375-10397

7. Henderson AMG, J. A.; Luukinen, B.; Buhl, K.; Stone, D., Glyphosate Technical Fact Sheet. National Pesticide Information Center, 2010

8. Sammons RD, Gruys KJ, Anderson KS, Johnson KA, Sikorski JA (1995) Reevaluating glyphosate as a transition-state inhibitor of EPSP synthase: identification of an EPSP synthase. cntdot. EPSP. cntdot. glyphosate ternary complex. Biochemistry 34:6433-6440

9. Boocock MR, Coggins JR (1983) Kinetics of 5-enolpyruvylshikimate3-phosphate synthase inhibition by glyphosate. FEBS Lett 154:127-133

10. Caetano MS, Ramalho TC, Botrel DF, da Cunha EFF, de Mello WC (2012) Understanding the inactivation process of organophosphorus herbicides: a DFT study of glyphosate metallic complexes with $\mathrm{Zn}^{2+}$, $\mathrm{Ca}^{2+}, \mathrm{Mg}^{2+}, \mathrm{Cu}^{2+}, \mathrm{Co}^{3+}, \mathrm{Fe}^{3+}, \mathrm{Cr}^{3+}$, and $\mathrm{Al}_{3}$. Int J Quantum Chem 112:2752-2762

11. Subramaniam V, Hoggard PE (1988) Metal complexes of glyphosate. J Agric Food Chem 36(6):1326-1329

12. Coutinho CFB, Mazo LH (2005) Complexos Metálicos Com O Herbicida Glifosato: Revisão. Quim Nova 26:1038-1045

13. Glass RL (1984) Metal complex formation by glyphosate. J Agric Food Chem 32:1249-1263

14. Sheals J, Persson P, Hedman B (2001) IR and EXAFS spectroscopic studies of glyphosate protonation and copper(II) complexes of glyphosate in aqueous solution. Inorg Chem 40(17):4302-4309

15. Tsui MT, Wang WX, Chu LM (2005) Influence of glyphosate and its formulation (Roundup) on the toxicity and bioavailability of metals to Ceriodaphnia dubia. Environ Pollut 138:59-68

16. Borggaard OK, Gimsing AL (2008) Fate of glyphosate in soil and the possibility of leaching to ground and surface waters: a review. Pest Manag Sci 64:441-456

17. Sihtmäe M, Blinova I, Künnis-Beres K, Kanarbik L, Heinlaan M, Kahru A (2013) Ecotoxicological effects of different glyphosate formulations. Appl Soil Ecol 72:215-224

18. Mesnage R, Defarge N, Spiroux de Vendômois J, Séralini GE (2014) Major pesticides are more toxic to human cells than their declared active principles. Biomed Res Int 2014:179691. https://doi. org/10.1155/2014/179691

19. Banks L, Kennedy AC, Kremer RJ, Eivasi F (2014) Soil microbial community response to surfactants and herbicides in two soils. Appl Soil Ecol 74:12-20

20. Cavalieri SD, Velini ED, Silva FML, São José AR, Andrade GJM (2012) Acúmulo de nutrientes e matéria seca na parte aérea de dois cultivares de soja RR sob efeito de formulações de glyphosate. Planta daninha 30:349-358

21. Feng PCC, Chiu T, Douglas Sammons R (2003) Glyphosate efficacy is contributed by its tissue concentration and sensitivity in velvet leaf (Abutilon theophrasti). Pestic Biochem Physiol 77:83-91

22. Tesfamariam T, Bott S, Cakmak I, Roemheld V, Neumann G (2009) Glyphosate in the rhizosphere-Role of waiting times and different glyphosate binding forms in soils for phytotoxicity to non-target plants. Eur J Agron 31:126-132

23. Kremer RJ, Means NE, Kim S (2005) Glyphosate effects soybean root exudation and rhizosphere micro-organisms. Int J Environ Anal Chem $85: 1165-1174$

24. Neumann G, Kohls S, Landsberg E, Souza S-OK, Yamada T, Römheld V (2006) Relevance of glyphosate transfer to non-target plants via the rhizosphere. J Plant Dis Prot 20:963-969

25. Laitinen P, Sari Remo S, Siimes K (2007) Glyphosate translocation from plants to soil-does this contribute a significant proportion of residues in soil? Plant Soil 300:51-60 
26. Monsanto, frequently asked questions, 2017. https://www.roundup.ca/ en/faq. Accessed 20 May 2017

27. Bott S, Tesfamariam T, Kania A, Eman B, Aslan N, Römheld VA, Neumann G (2011) Phytotoxicity of glyphosate soil residues re-mobilised by glyphosate fertilization. Plant Soil 342:249-263

28. Munira S, Farenhorst A, Flaten D, Grant C (2016) Phosphate fertilizer impacts on glyphosate sorption by soil. Chemosphere 153:471-477

29. Al-Rajab AJ, Amellal SA, Schiavon M (2008) Sorption and leaching of 14 C glyphosate in agricultural soils. Agron Sustain Dev 28:419-428

30. Simonsen L, Fomsgaard IS, Svensmark BA, Henrik Splid N (2008) Fate and availability of glyphosate and AMPA in agricultural soil. J Environ Sci Health Part B 43:365-375

31. Aparicio VC, De Gerónimo E, Marino D, Primost J, Carriquiriborde PA, Costa JL (2013) Environmental fate of glyphosate and aminomethylphosphonic acid in surface waters and soil of agricultural basins. Chemosphere 93:1866-1873

32. Coupe RH, Kalkhoff SJ, Capel PDA, Gregoire C (2012) Fate and transport of glyphosate and aminomethylphosphonic acid in surface waters of agricultural basins. Pest Manag Sci 68:16-30

33. Sasal MC, Demonte L, Cislaghi A, Gabioud EA, Oszust JD, Wilson MG, Michlig N, Beldoménico HR, Repetti MR (2015) Glyphosate loss by runoff and its relationship with phosphorus fertilization. J Agric Food Chem 63:4444-4448

34. Zablotowicz RMA, Reddy KN (2004) Impact of glyphosate on the Bradyrhizobium japonicum symbiosis with glyphosate-resistant transgenic soybean: a mini-review. J Environ Qual 33:825-831

35. Aristilde L, Reed ML, Wilkes RA, Youngster T, Kukurugya MA, Katz V, Sasaki CRS (2017) Glyphosate-induced specific and widespread perturbations in the metabolome of Soil Pseudomonas species. Front Environ Sci 5:162

36. Zobiole LH, Kremer RJ, de Oliveira RS Jr., Constantin J (2010), Glyphosate affects micro-organisms in rhizospheres of glyphosate-resistant soybeans. J Appl Microbiol 110:118-127

37. Kremer RJ, Means NE (2009) Glyphosate and glyphosate-resistant crop interactions with rhizosphere microorganisms. Eur J Agron 31:153-161

38. Druille M, Cabello MN, Omacini M, Golluscio RA (2013) Glyphosate reduces spore viability and root colonization of arbuscular mycorrhizal fungi. Appl Soil Ecol 64:99-103

39. Druille M, García-Parisi PA, Golluscio RA, Cavagnaro FP, Omacini M (2016) Repeated annual glyphosate applications may impair beneficial soil microorganisms in temperate grassland. Agr Ecosyst Environ 230:184-190

40. Nivelle E, Verzeaux J, Chabot A, Roger D, Spicher F, Lacoux J, NavaSaucedo JE, Catterou M, Tétu T (2017) Does nitrogen fertilization history affects short-term microbial responses and chemical properties of soils submitted to different glyphosate concentrations. PLoS One 12:e0178342

41. Powell JR, Swanton CJ (2008) A critique of studies evaluating glyphosate effects on diseases associated with Fusarium spp. Weed Res 48:307-318

42. Johal GS, Huber DM (2009) Glyphosate effects on diseases of plants. Eur J Agron 31:144-152

43. Newman MM, Hoilett N, Lorenz N, Dick RP, Liles MR, Ramsier C, Kloepper JW (2016) Glyphosate effects on soil rhizosphere-associated bacterial communities. Sci Total Environ 543:155-160

44. Johal GS, Rahe JE (1984) Effect of soil borne plant-pathogenic fungi on the herbicidal action of glyphosate on bean seedlings. Phytopathology 74:950-955

45. Lévesque CA, Rahe JE, Eaves DM (1992) The effect of soil heat treatment and microflora on the efficacy of glyphosate in seedlings. Weed Res 32:363-373

46. Sharon A, Amsellem Z, Gressel J (1992) Glyphosate suppression of an elicited defense response. Plant Physiol 98:654-659

47. Sanogo S, Yang XB, Scherm H (2000) Effects of herbicides on Fusarium solani f. sp. glycines and development of sudden death syndrome in glyphosate-tolerant soybean. Phytopathology 90:57-66

48. Rosenbaum KK, Miller GL, Kremer RJ, Bradley KW (2014) Interactions between glyphosate, Fusarium infection of common waterhemp (Amaranthus rudis), and soil microbial abundance and diversity in soil collections from Missouri. Weed Sci 62:71-82

49. Lynch JM, Penn DJ (1980) Damage to cereals caused by decaying weed residues. J Sci Food Agric 31:321-324
50. Smiley RW, Ogg AGJ, Cook RJ (1992) Influence of glyphosate on Rhizoctonia root rot, growth and yield of barley. Plant Dis 76:937-942

51. Kawate MK, Colwell SG, Ogg AGJ, Kraft JM (1997) Effect of glyphosatetreated henbit (Lamium amplexicaule) and downy brome (Bromus tectorum) on Fusarium solani f. sp. pisi and Pythium ultimum. Weed Sci 45:739-743

52. Sanogo S, Yang XB, Lundeen P (2001) Field response of glyphosatetolerant soybean to herbicides and sudden death syndrome. Plant Dis 85:773-779

53. Fernandez MR, Zentner RP, Basnyat P, Gehl D, Selles F, Huber D (2009) Glyphosate associations with cereal diseases caused by Fusarium spp. in the Canadian Prairies. Eur J Agron 31:133-143

54. Lévesque CA, Rahe JE, Eaves DM (1987) Effects of glyphosate on Fusarium spp.: its influence on root colonization of weeds, propagule density in the soil, and crop emergence. Can J Microbiol 33:354-360

55. Sharma-Poudyal D, Paulitz TC, du Toit $\perp$ (2016) Timing of glyphosate applications to wheat cover crops to reduce onion stunting caused by Rhizoctonia solani. Plant Dis 100:1474-1481

56. Acharya J, Bakker MG, Moorman TB, Kaspar TC, Lenssen AW, Robertson AE (2016) Time interval between cover crop termination and planting influences corn seedling disease, plant growth, and yield. Plant Dis 101:591-600

57. Newman MM, Lorenz N, Hoilett N, Lee NR, Dick RP, Liles MR, Ramsier C, Kloepper JW (2016) Changes in rhizosphere bacterial gene expression following glyphosate treatment. Sci Total Environ 553:32-41

58. Allegrini M, Zabaloy MC, Gómez EV (2015) Ecotoxicological assessment of soil microbial community tolerance to glyphosate. Sci Total Environ 533:60-68

59. Zabaloy MC, Gómez E, Garland JL, Gómez MA (2012) Assessment of microbial community function and structure in soil microcosms exposed to glyphosate. Appl Soil Ecol 61:333-339

60. De Maria N, Becerril JM, García-Plazaola JI, Hernández A, de Felipe MR (2006) New insights on glyphosate mode of action in nodular metabolism: role of shikimate accumulation. J Agric Food Chem 54:2621-2628

61. Nicolas V, Oestreicher N, Vélot C (2016) Multiple effects of a commercial Roundup ${ }^{\circledR}$ formulation on the soil filamentous fungus Aspergillus nidulans at low doses: evidence of an unexpected impact on energetic metabolism. Environ Sci Pollut Res Int 23:14393-14404

62. Olorunsogo OO, Bababunmi EA, Bassir O (1978) Uncouppling of corn mitochondria by N(phosphomehtyl)glycine. FEBS Lett 97:279-282

63. Swanson NL, Hoy J, Seneff S (2016) Evidence that glyphosate is a causative agent in chronic sub-clinical metabolic acidosis and mitochondrial dysfunction. Int J Hum Nutr Functl Mednal o 4:32

64. Liu CM, McLean PA, Sookdeo CC, Cannon FC (1991) Degradation of the herbicide glyphosate by members of the family Rhizobiaceae. Appl Environ Microbiol 57:1799-1804

65. Dick RE, Quinn JP (1995) Glyphosate-degrading isolates from environmental samples: occurrence and pathways of degradation. Appl Microbiol Biotech J 43:545-550

66. Krzysko-Lupika T, Orlick A (1997) The use of glyphosate as the sole source of phosphorus or carbon for the selection of soil borne strains capable to degrade this herbicide. Chemosphere 34:2601-2605

67. Gimsing AL, Borggaard OK, Jacobsen OS, Aamandb J, Sørensen J (2004) Chemical and microbiological soil characteristics controlling glyphosate mineralisation in Danish surface soils glyphosate mineralisation in Danish surface soils. Appl Soil Ecol 27:233-242

68. Castro JV, Peralba MCR, Ayub MAZ (2007) Biodegradation of the herbicide glyphosate by filamentous fungi in platform shaker and batch bioreactor. J Environ Sci Health 42:883-886

69. Mijangos I, Becerril JM, Albizu I, Epelde L, Garbisu C (2009) Effects of glyphosate on rhizosphere soil microbial communities under two different plant compositions by cultivation-dependent and -independent methodologies. Soil Biol Biochem 41:505-513

70. Hanley RL, Senseman SA, Hons RM, Zuberer DA (2000) Effects of glyphosate on soil microbial activity and biomass. Weed Sci 48:89-93

71. Liu LZK, Punja ZK, Rahe JE (1997) Altered root exudation and suppression of induced lignification as mechanisms of predisposition by glyphosate of bean roots (Phaseolus vulgaris L.) to colonization by Pythium spp. Physiol Mol Plant Pathol 51:111-127

72. Chaboussou F (2004) Healthy crops, a new agricultural revolution. Jon Carpenter Publishing, Charlbury 
73. Keen NT, Holliday MJ, Yoshikawa M (1982) Effects of glyphosate on glyceollin production and the expression of resistance to Phytophthora megasperma f. sp. glycinea in soybean. Phytopathol 72:1467-1470

74. Johal GS, Rahe JE (1988) Glyphosate, hypersensitivity and phytoalexin accumulation in the incompatible bean anthracnose host-parasite interaction*. Physiol Mol Plant Pathol 32:267-281

75. Lévesque CA, Rahe JE (1992) Herbicide interactions with fungal root pathogens, with special reference to glyphosate. Annu Rev Phytopathol 30:579-602

76. Brammal RA, Higgins VJ (1988) The effect of glyphosate on resistance of tomato to Fusarium crown and root rot disease and on the formation of host structural defensive barriers. Can J Bot 66:1547-1555

77. Cerdeira AL, Duke SO (2006) The current status and environmental impacts of glyphosate-resistant crops: a review. J Environ Qual 35:1633-1658

78. Larson RL, Hill A, Fenwick A, Kniss AR, Hanson LEA, Miller SD (2006) Influence of glyphosate on Rhizoctonia and Fusarium root rot in sugar beet. Pest Manag Sci 62:1182-1192

79. Zobiole LHS, Bonini EA, de Oliviera RS Jr., Kremer RJ, Ferrarese-Filho O (2010) Glyphosate affects lignin content and amino acid production in glyphosate-resistant soybean. Acta Physiol Plant 32:831-837

80. Fernandez MR, Zentner RP, DePauw RM, Gehl DTA, Stevenson FC (2007) Impacts of crop production factors associated with Fusarium head blight in barley in eastern Saskatchewan. Crop Sci 47:1574-1584

81. Dordas C (2008) Role of nutrients in controlling plant diseases in sustainable agriculture. A review. Agron Sustain Dev 28:33-46

82. Cakmak I, Yazici A, Tutus Y, Ozturk L (2009) Glyphosate reduced seed and leaf concentrations of calcium, manganese, magnesium, and iron in non-glyphosate resistant soybean. Eur J Agron 31:114-119

83. Eker S, Ozturk L, Yazici A, Erenoglu B, Romheld V, Cakmak I (2006) Foliarapplied glyphosate substantially reduced uptake and transport of iron and manganese in sunflower (Helianthus annuus L.) plants. J Agric Food Chem 54:10019-10025

84. Ozturk L, Yazici A, Eker S, Gokmen O, Römheld VA, Cakmak I (2008) Glyphosate inhibition of ferric reductase activity in iron deficient sunflower roots. New Phytol 177:899-906

85. Bernards ML, Thelen KD, Penner D (2005) Glyphosate efficacy is antagonized by manganese. Weed Technol 19:27-34

86. Bailey WA, Poston DH, Wilson HP, Hines TE (2002) Glyphosate interactions with manganese 1. Weed Technol 16:792-799

87. Thelen K, Jackson EP, Penner D (1995) The basis for the hard-water antagonism of glyphosate activity. Weed Sci 43:541-548

88. de Andrade GJM, Rosolem CA (2011) Uptake of manganese in $\mathrm{Rr}$ soybean under glifosate application. R Bras Ci Solo 335:961-968

89. Rosolem CA, de Andrade GAM, Lisboa IP, Zoca SM (2010) manganese uptake and redistribution in soybean as affected by glyphosate. R Bras Ci Solo 34:1915-1922

90. Duke SO, Reddy KN, Bu K, Cizdziel JV (2012) Effects of glyphosate on the mineral content of glyphosate-resistant soybeans (Glycine max). J Agric Food Chem. 60(27):6764-6771
91. Duke SO, Rimando AM, Reddy KN, Cizdziel JV, Bellaloui N, Shaw DR, Williams II MM, Maul JE (2017) Lack of transgene and glyphosate effects on yield, and mineral and amino acid content of glyphosate-resistant soybean. Pest Manag Sci. https://doi.org/10.1002/ps.4625

92. Petter FA, Zuffo AM, Neto FA, Pacheco LP, de Almeida FA, Andrade FR, Junior JMZ (2016) Effect of glyphosate and water stress on plant morphology and nutrient accumulation in soybean. AJCS 10:251-257

93. Serra AP, Marchetti ME, da Silva C, Dias RC, Christoffoleti PJ (2011) Glyphosate influence on nitrogen, manganese, iron, copper and zinc nutritional efficiency in glyphosate resistant soybean. Ciência Rural Santa Maria 41:77-84

94. Zobiole LHS, de Oliviera RS Jr., Huber D, Constantin J, de Castro C, de Oliviera FA, de Oliviera A (2010) Glyphosate reduces shoot concentrations of mineral nutrients in glyphosate resistant soybeans. Plant Soil 328:57-69

95. Zobiole LHS, Kremer RJ, OJR S, Constantin J (2011) Glyphosate affects chlorophyll, nodulation and nutrient accumulation of "second generation" glyphosate-resistant soybean (Glycine max L.). Pestic Biochem Physiol 99:53-60

96. Bott S, Tesfamariam T, Candan H, Cakmak I, Römheld V, Neumann G (2008) Glyphosate-induced impairment of plant growth and micronutrient status in glyphosate-resistant soybean (Glycine max L.). Plant Soil 312:185-194

97. Zobiole LHS, Junior RSO, Kremer RJ (2010) Nutrient accumulation and photosynthesis in glyphosate-resistant soybeans is reduced under glyphosate use. J Plant Nutr 33:1860-1873

98. Reddy KN, Riimando AM, Duke SO (2004) Aminomethylphosphonic acid, a metabolite of glyphosate, causes injury in glyphosate-treated, glyphosate-resistant soybean. J Agric Food Chem 52:5139-5143

99. Ding W, Reddy K, Zablotowicz RM, Bellaloui N, Arnold Bruns H (2011) Physiological responses of glyphosate resistant and glyphosatesensitive soybean to aminomethylphosphonic acid, a metabolite of glyphosate. Chemosphere 83:593-598

100. Gomes MP, Smedbol E, Chalifour A, Hénault-Ethier L, Labrecque M, Lepage L, Lucotte M, Juneau P (2014) Alteration of plant physiology by glyphosate and its by-product aminomethylphosphonic acid: an overview. J Exp Bot 65:4691-4703

101. Zobiole L, Kremer R, de Oliveira RS Jr., Constantin J (2012) Glyphosate effects on photosynthesis, nutrient accumulation, and nodulation in glyphosate-resistant soybean. Z Pflanzenernähr Bodenk 175:319-330

102. Mesnage R, Defarge N, de Vendômois JS, Séralini GE (2015) Potential toxic effects of glyphosate and its commercial formulations below regulatory limits. Food Chem Toxicol 84:133-153

103. Samsel A, Seneff S (2016) Glyphosate pathways to modern diseases V: amino acid analogue of glycine in diverse proteins. JBPC 16:9-46 